\title{
The prognostic value of postoperative tumor marker conversion for gastric cancer
}

\author{
Jeongju Noh', Ki Bum Park²,3 Oh Kyoung Kwon ${ }^{2,3}$ \\ ${ }^{1}$ Department of Surgery, Kyungpook National University Hospital, Daegu; \\ ${ }^{2}$ Department of Surgery, School of Medicine, Kyungpook National University, Daegu; \\ ${ }^{3}$ Gastric Cancer Center, Kyungpook National University Chilgok Hospital, Daegu, Korea
}

Purpose: Preoperative positivity of serum tumor markers has been associated with poor long-term survival among patients with gastric cancer. However, there have been a considerable number of patients who have experienced a normalization of tumor markers (negative conversion) after curative treatment. This study aimed to evaluate the correlation between postoperative tumor marker conversion and survival after gastrectomy among gastric carcinoma patients.

Methods: We analyzed the clinical data of 129 patients who underwent curative gastrectomy with elevated preoperative carcinoembryonic antigen or carbohydrate antigen 19-9 between January 2009 and December 2013.

Results: Positive tumor markers converted to negative markers 6 months after surgery in 91 patients (70.5\%). The patients with a negative conversion of tumor markers had significantly better outcomes than those without negative conversion (overall survival [OS] 63.9 months vs. 41.1 months, $\mathrm{P}<0.001$; disease-free survival [DFS] 59.3 months vs. 33.2 months, $\mathrm{P}<0.001$ ). Multivariate analyses showed that tumor marker conversion and lymph node metastasis were independent predictors of OS and DFS. During follow-up, tumor markers became elevated again (positive reconversion) in 23 patients (25.3\%), with a negative conversion of tumor markers at 6 months after gastrectomy. Among the patients with a positive reconversion of tumor markers, gastric cancer recurred in 18 patients (78.3\%).

Conclusion: Negative tumor marker conversion after curative gastrectomy strongly predicts a better prognosis. Patients without negative tumor marker conversion and those with positive reconversion after normalization should be carefully monitored because of the high possibility of recurrence.

Keywords: Carcinoembryonic antigen, CA 19-9 antigen, Survival

\section{INTRODUCTION}

Tumor markers are defined as circulating substances that can be measured quantitatively and that have a potential causal or other association with malignant neoplasia [1,2]. Because tumor markers indicate the cellular, biochemical, molecular, and genetic alter-

Received: Jun 27, 2020 Revised: Sep 20, 2020 Accepted: Oct 19, 2020

Correspondence to: Oh Kyoung Kwon

Gastric Cancer Center, Kyungpook National University Chilgok Hospital,

807 Hoguk-ro, Buk-gu, Daegu 41404, Korea

Tel: +82-53-200-2709, Fax: +82-53-200-2027

E-mail: okugisurg@knu.ac.kr

ORCID: Jeongju Noh (https://orcid.org/0000-0002-0323-617X), Ki Bum Park (https:// orcid.org/0000-0001-5404-5667), Oh Kyoung Kwon (https://orcid.org/0000-00023614-8563)

Copyright (C) 2020 Korean Society of Surgical Oncology

This is an Open Access article distributed under the terms of the Creative Commons Attribution Non-Commercial License (http://creativecommons.org/licenses/by-nc/4.0) which permits unrestricted non-commercial use, distribution, and reproduction in any medium, provided the original work is properly cited. ations caused by cancer, they have been utilized for the early diagnosis of cancer, postoperative surveillance, prognosis assessment, therapy prediction, and to monitor responses to systemic therapy [1,3-5]. Currently, there are no specific tumor markers for gastric cancer. The commonly used markers for gastric cancer are carcinoembryonic antigen (CEA) and carbohydrate antigen (CA) 19-9.

After curative gastric cancer surgery, computed tomography (CT), and esophagogastroduodenoscopy (EGD) are the primary methods used to evaluate recurrence. Although tumor markers are not sufficient in terms of sensitivity and specificity [6], most surgeons still use tumor markers to monitor recurrence after gastrectomy because they are easily measured postoperatively by blood tests during outpatient follow-up.

Preoperative positivity of serum tumor markers has been associated with poor long-term survival among gastric cancer patients [7-10]. Previous studies have demonstrated that the determination of preoperative serum levels of both CEA and CA 19-9 has provided additional prognostic information for gastric cancer patients 
who have undergone gastrectomy. Patients with preoperative positivity for one of these tumor markers have a high risk of recurrence, even in the early stages of gastric cancer [9]. However, many patients have experienced a normalization of preoperatively elevated tumor markers (negative conversion) after curative gastrectomy, and the clinical impact of negative conversion has not been well evaluated. Furthermore, few studies have investigated the implications of positive reconversion after initial negative conversion $[11,12]$. This study aimed to evaluate the correlation between postoperative tumor marker conversion and survival after gastrectomy for gastric carcinoma.

\section{METHODS}

The Institutional Review Board (IRB) of Kyungpook National University Chilgok Hospital approved this study (IRB No. 201601-017). Written informed consent was waived by the IRB. Preoperative serum CEA and CA 19-9 levels were measured in 2,706 patients with gastric adenocarcinoma at Kyungpook National University Hospital and Kyungpook National University Chilgok Hospital, Daegu, Republic of Korea, between January 2009 and December 2013, and 258 patients (9.8\%) who had elevated preoperative CEA or CA 19-9 levels were investigated. We excluded 129 patients: those who underwent noncurative surgery, patients with remnant gastric cancer, patients without follow-up tumor marker test results, patients who received neoadjuvant chemotherapy, patients who had malignancies in other organs, and patients with pulmonary disease or liver disease. Finally, the remaining 129 patients were included in the study - all of the patients who underwent curative distal gastrectomy or total gastrectomy with D2 lymph node dissection.

The serum CEA and CA 19-9 were measured by radioimmunoassay analysis using CEA-RIACT and ELSA-CA19-9 (Cisbio Bioassays, Codolet, France). The cutoff values were $7 \mathrm{ng} / \mathrm{mL}$ for CEA and $37 \mathrm{U} / \mathrm{mL}$ for CA 19-9 according to manufacturer's instructions. Negative conversion of the tumor markers was defined as the normalization of either of the markers, CEA or CA 19-9, which was assessed 6 months after curative gastrectomy. Positive reconversion was defined as the re-elevation of one of the markers, CEA or CA 19-9, after initial negative conversion.

Follow-up examinations were performed every 3 months for the first year, every 6 months for the next 2 years, and every year thereafter. The follow-up program included a clinical examination, hematological analyses, tumor marker assays (at each check-up), CT (every 6 months), and EGD (once a year).

Chi-square tests and Fisher exact tests were used to analyze clinicopathologic features. Disease-free survival (DFS) and overall survival (OS) were calculated by the Kaplan-Meier method and compared using the log-rank test. Variables that significantly affected DFS and OS were investigated by multivariate analysis using Cox regression. P-values $<0.05$ were considered statistically significant.

\section{RESULTS}

The mean post-gastrectomy follow-up period was 35.1 months. Before gastrectomy, CEA was elevated in 45 patients (34.9\%), CA 19-9 was elevated in 98 patients (76.0\%), and both tumor markers were elevated in 14 patients (10.9\%). The positive tumor markers converted to negative markers 6 months after surgery in 91 patients (70.5\%). The correlation between tumor marker conversion and clinicopathological variables is presented in Table 1. Tumor marker conversion was significantly related to the depth of invasion and tumor stage. Among patients without negative conversion, there were more patients with $\mathrm{T} 1$ gastric cancer, and their tumors tended to be smaller. We analyzed the correlation between tumor marker conversion and other variables, particularly among patients with tumors $<5 \mathrm{~cm}$ in diameter and those with $\mathrm{T} 1$ gastric cancer. Tumor marker conversion was associated with American Society of Anesthesiologists (ASA) score among patients with tumor diameters $<5 \mathrm{~cm}(\mathrm{P}=0.060)$ and patients with $\mathrm{T} 1$ gastric cancer $(\mathrm{P}=0.019)$.

During follow-up, tumor recurrence was detected in 46 patients (35.7\%), including two patients who had been cured of early gastric cancer and 44 patients who had been cured of advanced gastric cancer. The mean period of recurrence was 13.5 months (range, 2.6-35.2 months). Locoregional recurrence was observed in six patients (13.0\%), hematogenous recurrence in nine patients (19.6\%), distant lymph node recurrence in eight patients (17.4\%), peritoneal recurrence in 14 patients (30.4\%), and combined recurrences in nine patients (19.6\%). There was no significant difference in the recurrence patterns between patients with tumor marker conversion and those without conversion. Additionally, there were no significant differences in recurrence pattern according to the positivity of CEA, CA 19-9, or both tumor markers. However, peritoneal recurrence was significantly associated with CA 19-9 positivity $(\mathrm{P}=0.036)$, and peritoneal recurrence trended toward an association with negative tumor marker conversion $(\mathrm{P}=0.084)$.

Fig. 1 shows survival distributions according to tumor marker conversion. Patients who experienced tumor marker conversion had significantly better outcomes than those who did not experience conversion (OS 63.9 months vs. 41.1 months, $\mathrm{P}<0.001$; DFS 59.3 months vs. 33.2 months, $\mathrm{P}<0.001)$. Tumor recurrence occurred within 15 postoperative months in 19 patients (79.2\%) who 
Table 1. Correlation between tumor marker conversion and clinicopathological variables

\begin{tabular}{|c|c|c|c|c|}
\hline \multirow{2}{*}{ Variable } & \multirow{2}{*}{ All patients $(n=129)$} & \multicolumn{2}{|c|}{ Tumor marker conversion } & \multirow{2}{*}{ P-value } \\
\hline & & Yes $(n=91)$ & No $(n=38)$ & \\
\hline Age (yr) & $63.0 \pm 11.5$ & $61.0 \pm 12.4$ & $63.5 \pm 12.0$ & 0.416 \\
\hline Sex & & & & 0.785 \\
\hline Male & $86(66.7)$ & $60(65.9)$ & $26(68.4)$ & \\
\hline Female & 43 (33.3) & $31(34.1)$ & $12(31.6)$ & \\
\hline ASA & & & & 0.418 \\
\hline 1 & $67(51.9)$ & $48(52.7)$ & $19(50.0)$ & \\
\hline 2 & $61(47.3)$ & 43 (47.3) & $18(47.4)$ & \\
\hline 3 & $1(0.8)$ & 0 & $1(2.6)$ & \\
\hline CEA (ng/mL) & $12.0 \pm 26.7$ & $9.1 \pm 13.9$ & $14.7 \pm 36.5$ & 0.812 \\
\hline CA 19-9 (U/mL) & $154 \pm 329.9$ & $134.0 \pm 306.0$ & $173.0 \pm 231.7$ & 0.258 \\
\hline Pathologya) & & & & 0.993 \\
\hline Differentiated & $51(39.5)$ & $36(39.6)$ & 15 (39.5) & \\
\hline Undifferentiated & $78(60.5)$ & $55(60.4)$ & $23(60.5)$ & \\
\hline Lauren classification & & & & 0.765 \\
\hline Intestinal & 45 (34.9) & $31(34.1)$ & $14(36.8)$ & \\
\hline Diffuse & 66 (51.2) & $46(50.5)$ & $20(52.6)$ & \\
\hline Mixed & $18(14.0)$ & $14(15.4)$ & $4(10.5)$ & \\
\hline Tumor size $(\mathrm{cm})$ & $5.2 \pm 3.1$ & $5.6 \pm 3.3$ & $4.4 \pm 2.5$ & 0.064 \\
\hline Depth of invasion & & & & 0.049 \\
\hline $\mathrm{T} 1$ & 27 (20.9) & $15(16.5)$ & $12(31.6)$ & \\
\hline $\mathrm{T} 2$ & $13(10.1)$ & $10(11.0)$ & $3(7.9)$ & \\
\hline T3 & $40(31.0)$ & $34(37.4)$ & $6(15.8)$ & \\
\hline $\mathrm{T} 4$ & 49 (38.0) & $32(35.2)$ & $17(44.7)$ & \\
\hline No. of positive LNs & $6.3 \pm 9.2$ & $5.8 \pm 8.3$ & $8.3 \pm 11.8$ & 0.391 \\
\hline Total No. of dissected LNs & $37.8 \pm 16.1$ & $39.5 \pm 16.9$ & $39.8 \pm 13.9$ & 0.527 \\
\hline Stage (AJCC 7th ed) & & & & 0.010 \\
\hline I & $28(21.7)$ & $15(16.5)$ & $13(34.2)$ & \\
\hline$\|$ & $31(24.0)$ & $28(30.8)$ & $3(7.9)$ & \\
\hline III & $67(51.9)$ & $46(50.5)$ & $21(55.3)$ & \\
\hline N & $3(2.3)$ & $2(2.2)$ & $1(2.6)$ & \\
\hline Adjuvant chemotherapy & $80(62.0)$ & $60(65.9)$ & $20(52.6)$ & 0.156 \\
\hline
\end{tabular}

Values are presented as mean \pm standard deviation or number (\%).

ASA, American Society of Anesthesiologists; CEA, carcinoembryonic antigen; CA, carbohydrate antigen; LN, lymph node; AJCC, American Joint Committee on Cancer.

${ }^{a}$ Differentiation type was divided according to the degree of glandular differentiation in the Japanese classification.

did not experience tumor marker conversion, and in five patients (20.8\%) after a longer postoperative interval. Among patients who did experience tumor marker conversion, 12 (54.5\%) had tumor recurrence within 15 postoperative months. Survival distributions according to tumor marker conversion in each tumor marker are shown in Fig. 2. OS and DFS for CEA conversion and non-conversion were 61.4 months versus 21.6 months $(\mathrm{P}<0.001)$ and 42.4 months versus 13.5 months $(\mathrm{P}<0.001)$, respectively. OS and DFS for CA 19-9 conversion and non-conversion were 66.0 months versus 44.3 months $(\mathrm{P}<0.001)$ and 62.6 months versus 35.0 months $(\mathrm{P}<0.001)$, respectively.

Table 2 shows the results of the univariate and multivariate analyses of prognostic factors. Univariate analysis showed that CEA level, tumor marker conversion, tumor size, depth of invasion, and lymph node metastasis were significant predictors of OS. Tumor marker conversion, depth of invasion, and lymph node metastasis were independent predictors of OS according to the multivariate analysis. Predictors of DFS in the univariate analysis were CEA level, tumor marker conversion, tumor size, depth of invasion, and lymph node metastasis. Multivariate analysis revealed that tumor 
marker conversion, CEA level, and lymph node metastasis were statistically significant predictors of DFS.

Among the patients with negative tumor marker conversion 6 months after gastrectomy, tumor markers became elevated again (positive reconversion) in 23 patients (25.3\%) during follow-up.
Among those who experienced positive reconversion, gastric cancer recurred in 18 patients (78.3\%). Regarding baseline clinicopathologic characteristics, there were no significant differences between patients with tumor marker reconversion and those without reconversion. The survival distributions of patients according to
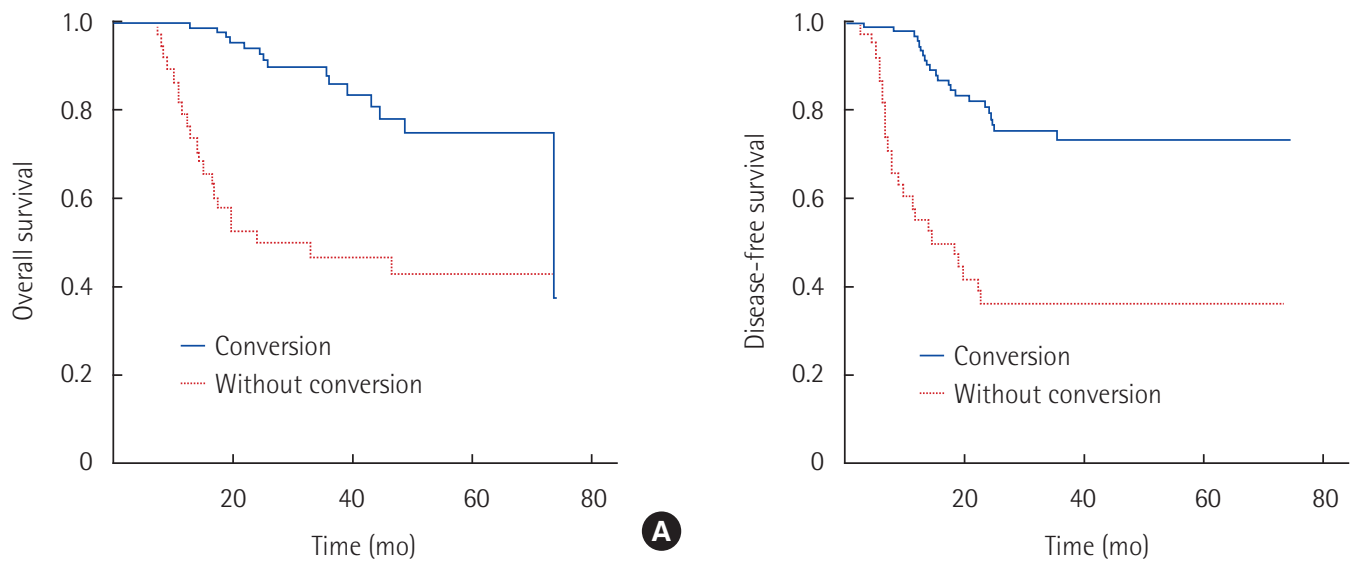

Fig. 1. Survival distributions according to tumor marker conversion status. (A) Overall survival (63.9 months vs. 41.1 months, $P<0.001)$. (B) Disease-free survival (59.3 months vs. 33.2 months, $\mathrm{P}<0.001$ ).

Table 2. Results of univariate and multivariate analyses of prognostic factors

\begin{tabular}{|c|c|c|c|c|c|c|c|c|}
\hline \multirow{3}{*}{ Variable } & \multicolumn{4}{|c|}{ OS } & \multicolumn{4}{|c|}{ DFS } \\
\hline & \multirow{2}{*}{$\begin{array}{c}\text { Univariate } \\
\text { P-value }\end{array}$} & \multicolumn{3}{|c|}{ Multivariate } & \multirow{2}{*}{$\begin{array}{c}\text { Univariate } \\
\text { P-value }\end{array}$} & \multicolumn{3}{|c|}{ Multivariate } \\
\hline & & $H R$ & $95 \% \mathrm{Cl}$ & P-value & & HR & $95 \% \mathrm{Cl}$ & P-value \\
\hline Age ( $\geq 64 \mathrm{yr})$ & 0.590 & & & & 0.439 & & & \\
\hline Sex & 0.170 & & & & 0.330 & & & \\
\hline ASA & 0.594 & & & & 0.252 & & & \\
\hline \multicolumn{9}{|l|}{ Tumor marker levels } \\
\hline CEA ( $\geq 7 \mathrm{ng} / \mathrm{mL})$ & 0.028 & & & 0.271 & 0.008 & 1.921 & $1.066-3.461$ & 0.030 \\
\hline CA $19-9$ ( $\geq 37 \mathrm{U} / \mathrm{mL})$ & 0.064 & & & 0.178 & 0.059 & & & 0.491 \\
\hline Tumor marker conversion & $<0.001$ & 7.501 & $3.681-15.287$ & $<0.001$ & $<0.001$ & 8.213 & $4.241-15.906$ & $<0.001$ \\
\hline \multicolumn{9}{|l|}{ Pathology } \\
\hline Undifferentiated & 0.395 & & & & 0.250 & & & \\
\hline Lauren classification & 0.644 & & & & 0.828 & & & \\
\hline Tumor size $(\geq 5 \mathrm{~cm})$ & 0.003 & & & 0.877 & 0.003 & & & 0.868 \\
\hline Depth of invasion & $<0.001$ & & & 0.016 & $<0.001$ & & & 0.224 \\
\hline $\mathrm{T} 1$ & & 1.000 & & & & & & \\
\hline $\mathrm{T} 2$ & & 3.246 & $0.280-37.630$ & & & & & \\
\hline T3 & & 3.014 & $0.300-30.302$ & & & & & \\
\hline T4 & & 9.093 & $0.931-88.834$ & & & & & \\
\hline Lymph node metastasis & $<0.001$ & & & 0.010 & $<0.001$ & & & $<0.001$ \\
\hline No & & 1.000 & & & & 1.000 & & \\
\hline N1 & & 5.455 & $0.527-56.428$ & & & 9.883 & $1.103-88.536$ & \\
\hline N2 & & 10.839 & 1.117-105.129 & & & 52.585 & $6.774-408.182$ & \\
\hline N3 & & 15.495 & $1.612-148.926$ & & & 69.985 & $9.159-534.757$ & \\
\hline
\end{tabular}

OS, overall survival; DFS, disease-free survival; HR, hazard ratio; $\mathrm{Cl}$, confidence interval; $\mathrm{ASA}$, American Society of Anesthesiologists; $C E A$, carcinoembryonic antigen; $C A$, carbohydrate antigen. 
CEA

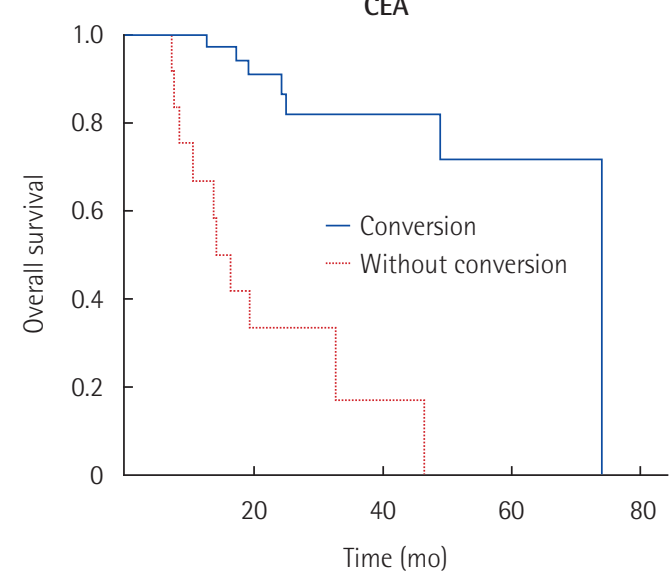

CA 19-9

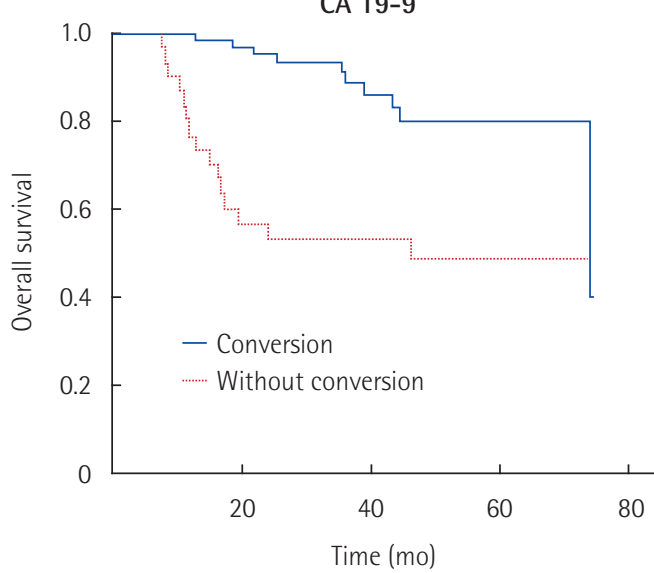

CEA

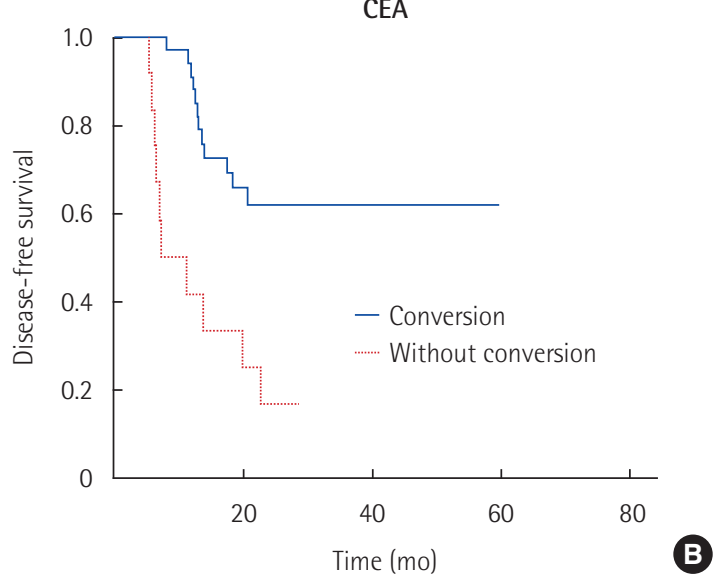

CA 19-9

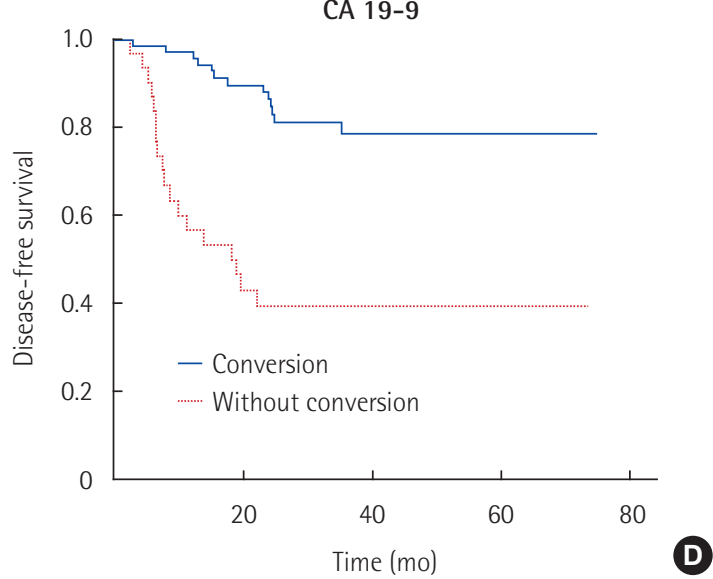

Fig. 2. Survival distributions according to tumor marker conversion status for each tumor marker. (A) Overall survival according to carcinoembryonic antigen (CEA) conversion status (61.4 months vs. 21.6 months, $\mathrm{P}<0.001$ ). (B) Disease-free survival according to CEA conversion status (42.4 months vs. 13.5 months, $P<0.001$ ). (C) Overall survival according to carbohydrate antigen (CA) 19-9 conversion status (66.0 months vs. 44.3 months, $P<0.001$ ). (D) Disease-free survival according to $C A 19-9$ conversion status (62.6 months vs. 35.0 months, $P<0.001$ ).
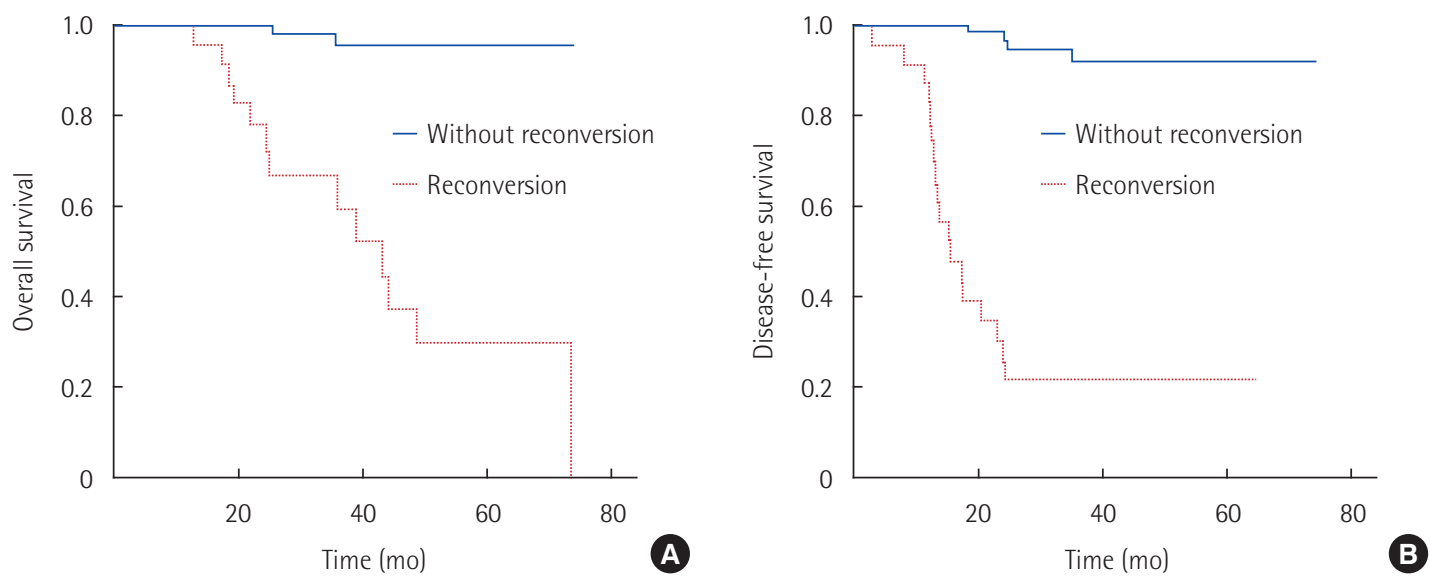

Fig. 3. Survival distributions according to tumor marker reconversion status. (A) Overall survival (72.3 months vs. 44.4 months, $P<0.001)$. (B) Disease-free survival (70.5 months vs. 25.8 months, $\mathrm{P}<0.001)$. 
tumor marker reconversions are shown in Fig. 3. Patients with tumor marker reconversion had significantly worse outcomes than those without reconversion (OS 72.3 months vs. 44.4 months, $\mathrm{P}<0.001$; DFS 70.5 months vs. 25.8 months, $\mathrm{P}<0.001$ ).

\section{DISCUSSION}

Serum CEA or CA 19-9 levels are often measured preoperatively in gastric cancer patients. CEA is an oncofetal protein that is normally present during fetal life but can be present in low concentrations in healthy adults [4]. Borderline levels occur with benign disorders, such as inflammatory bowel disease, pancreatitis, cirrhosis, and chronic obstructive pulmonary disease. The main clinical use of CEA is for patients with cancer of the colon and rectum. However, elevated CEA levels are observed not only in association with these tumors but also with carcinoma of the breast, lung, stomach, pancreas, uterine cervix, bladder, thyroid, and liver [1,4]. CA 19-9 is an incomplete glycolipid antigen of the Lewis blood group and is widely used as a serum marker for pancreatic cancer [13]. High levels of CA 19-9 can be observed in patients with other cancers, including those of the biliary tree, stomach, colon, liver, and lung. CA 19-9 is also elevated in conditions such as benign biliary tract disease, liver cirrhosis, and pancreatitis $[1,4,14]$. Concerning gastric cancer, these markers have limited clinical utility because of their low sensitivity and specificity [6]. When evaluating the prognostic value of tumor marker conversion, this low specificity must be considered. Thus, patients with malignant tumors of other organs were excluded from this study. We also excluded patients with pulmonary disease or liver disease, unlike previous studies [712,14-16].

Preoperative positivity of serum tumor markers has been associated with poor long-term survival among gastric cancer patients [710]. In a recent meta-analysis [6], preoperative positivity rates were $21.1 \%$ (range, 16\%-68\%) for CEA and 27.8\% (range, 14\%-68\%) for CA 19-9. CEA was known to be strongly associated with T category, $\mathrm{N}$ category, $\mathrm{M}$ category, and tumor stage. Maehara et al. [16] reported that CEA-positive patients had larger tumors, greater serosal invasion, more frequent lymphatic and vascular involvement, less expansive tumor growth, and higher rates of lymph node and hepatic metastases than CEA-negative patients. CA 19-9 has been associated with depth of invasion, lymph node metastasis, peritoneal metastasis, and stage [6]. In this study, the preoperative positivity rates of CEA and CA 19-9 were 3.7\% and 7.3\%, respectively. These low positivity rates could be because we included patients selected for surgical resection and those with a high incidence of early gastric cancer and used different cutofflevels of CEA.

In this study, we demonstrated that patients with postoperative tumor marker conversion had significantly better outcomes than those without conversion. Multivariate analysis showed that tumor marker conversion was a strong predictor of OS and DFS. Nam et al. [15] analyzed the implications of early tumor marker responses, namely elevation before surgery and normalization within 1 to 2 months after curative gastrectomy. Both OS and DFS were significantly longer among patients with a tumor marker response than among those without a response. These findings are similar to ours, except for the differing postoperative intervals. In clinical practice, negative tumor marker conversion can occur at different times after curative gastrectomy, even among patients with the same stage of cancer. In our study sample, 10 patients (7.8\%) received early postoperative intraperitoneal chemotherapy plus adjuvant systemic chemotherapy and more than half (64.5\%) of the patients with gastric cancer of stage II or higher received adjuvant systemic chemotherapy. To examine the changes in tumor markers according to multimodal treatment including curative gastrectomy, we tested the serum level of tumor markers at 6 months after curative surgery.

The levels of both CEA and CA 19-9 generally correlate with the tumor burden because they are shed from the expanding neoplasm [4]. It seems to be inevitable that tumor marker positivity will convert to negative after curative surgery. However, in this study, positive tumor markers were continuously elevated in 38 patients (29.5\%). One explanation may be the presence of micrometastases. Another explanation may be the differences in the underlying disease. In the group without negative conversion, there were more patients with T1 gastric cancer, and tumors tended to be smaller. Tumor marker conversion was related to ASA score among patients with tumor diameters $<5 \mathrm{~cm}$ and patients with $\mathrm{T} 1$ gastric cancer.

Elevated tumor marker levels detected during the follow-up of clinically disease-free patients often represent the first sign of relapse $[6,12]$. These findings may facilitate early clinical decision-making regarding the use of additional diagnostic methods - such as positron emission tomography and laparoscopy and the selection of appropriate chemotherapeutic regimens, which may contribute to prolonged survival for patients with recurrent gastric cancer [11]. In cases of localized recurrence, debulking surgery and intraperitoneal chemotherapy — such as early postoperative intraperitoneal chemotherapy and hyperthermic intraperitoneal chemotherapy for peritoneal metastases or locoregional chemotherapy through the hepatic artery for liver metastases — may be indicated $[17,18]$. Serial measurement during postoperative follow-up is particularly important for patients with high preoperative tumor marker levels. Takahashi et al. [12] reported that more than $90 \%$ of patients with elevated preoperative 
CEA levels had increased CEA levels again at the time of recurrence and that CA 19-9 levels increased again at recurrence in more than $90 \%$ of patients with high preoperative levels of this marker. In our study, tumor markers increased again (positive reconversion) in 23 (25.3\%) patients with negative tumor marker conversion. Among these patients, gastric cancer recurred in 18 patients (78.3\%), and patients with tumor marker reconversion had significantly worse outcomes than those without reconversion. Choi et al. [14] reported that the majority (90\%) of cases with recurrence to the liver had an elevated CEA level, whereas an elevated postoperative CA 19-9 level was more predictive of a peritoneal recurrence $(78.9 \%)$. In our results, there were no significant differences in the patterns of recurrence according to the positivity of CEA, CA 19-9, or both tumor markers ( $\mathrm{P}=0.174)$. However, we found that peritoneal recurrence was significantly associated with CA 19-9 positivity, and there was a trend toward an association between peritoneal recurrence and tumor marker conversion. Takahashi et al. [12] observed that patients with higher preoperative tumor marker levels were more likely to have a longer lead time than those with lower preoperative tumor marker levels. In this study, recurrence and tumor marker positivity were diagnosed simultaneously in 11 patients (61.1\%). These results indicate that monitoring for recurrence using tumor markers is useful but not sufficient. We need to recognize that positive tumor marker conversion can be assessed much later than the detection of recurrence by imaging in some patients [12].

The limitations of this study include the retrospective design and the small sample size with selection bias. Also, the low tumor marker specificity is one of the weak points of our study. Despite these limitations, our study showed that the combined detection of CEA and CA 19-9 provides prognostic information for gastric cancer after multimodal treatment, particularly among patients with high preoperative levels of these markers. Negative tumor marker conversion after curative gastrectomy strongly predicts a better prognosis. Patients without negative tumor marker conversion and those with positive reconversion after normalization should be carefully monitored due to the high possibility of recurrence. As such, physicians need to consider tumor marker conversion status during early clinical decision-making.

\section{CONFLICT OF INTEREST}

No potential conflict of interest relevant to this article was reported.

\section{ACKNOWLEDGMENTS}

This research was partly supported by the promotion project for regional advanced components and materials (A000600028) of the Ministry of Knowledge Economy, Korea.

\section{REFERENCES}

1. Seregni E, Ferrari L, Martinetti A, Bombardieri E. Diagnostic and prognostic tumor markers in the gastrointestinal tract. Semin Surg Oncol 2001;20:147-66.

2. Sikaroodi M, Galachiantz Y, Baranova A. Tumor markers: the potential of "omics" approach. Curr Mol Med 2010;10:249-57.

3. Duffy MJ. Role of tumor markers in patients with solid cancers: a critical review. Eur J Intern Med 2007;18:175-84.

4. Sabiston DC, Townsend CM. Tumor biology and tumor markers. In: Sabiston DC, Townsend CM, editors. Sabiston textbook of surgery: the biological basis of modern surgical practice. 19th ed. Philadelphia, PA: Elsevier Saunders; 2012. p. 692-719.

5. Duffy MJ. Tumor markers in clinical practice: a review focusing on common solid cancers. Med Princ Pract 2013;22:4-11.

6. Shimada H, Noie T, Ohashi M, Oba K, Takahashi Y. Clinical significance of serum tumor markers for gastric cancer: a systematic review of literature by the Task Force of the Japanese Gastric Cancer Association. Gastric Cancer 2014;17:26-33.

7. Ikeda Y, Oomori H, Koyanagi N, Mori M, Kamakura T, Minagawa $\mathrm{S}$, et al. Prognostic value of combination assays for CEA and CA 19-9 in gastric cancer. Oncology 1995;52:483-6.

8. Kodera Y, Yamamura Y, Torii A, Uesaka K, Hirai T, Yasui K, et al. The prognostic value of preoperative serum levels of CEA and CA19-9 in patients with gastric cancer. Am J Gastroenterol 1996; 91:49-53.

9. Marrelli D, Roviello F, De Stefano A, Farnetani M, Garosi L, Messano A, et al. Prognostic significance of CEA, CA 19-9 and CA 72-4 preoperative serum levels in gastric carcinoma. Oncology 1999;57:55-62.

10. Nakane Y, Okamura S, Akehira K, Boku T, Okusa T, Tanaka K, et al. Correlation of preoperative carcinoembryonic antigen levels and prognosis of gastric cancer patients. Cancer 1994;73:2703-8.

11. Komatsu S, Ichikawa D, Nishimura Y, Kubota T, Okamoto K, Shiozaki A, et al. Better outcomes by monitoring tumour dynamics using sensitive tumour markers in patients with recurrent gastric cancer. Anticancer Res 2013;33:1621-7.

12. Takahashi Y, Takeuchi T, Sakamoto J, Touge T, Mai M, Ohkura H, et al. The usefulness of CEA and/or CA19-9 in monitoring for recurrence in gastric cancer patients: a prospective clinical study. Gastric Cancer 2003;6:142-5. 
13. Ballehaninna UK, Chamberlain RS. The clinical utility of serum CA 19-9 in the diagnosis, prognosis and management of pancreatic adenocarcinoma: an evidence based appraisal. J Gastrointest Oncol 2012;3:105-19.

14. Choi SR, Jang JS, Lee JH, Roh MH, Kim MC, Lee WS, et al. Role of serum tumor markers in monitoring for recurrence of gastric cancer following radical gastrectomy. Dig Dis Sci 2006;51:2081-6.

15. Nam DH, Lee YK, Park JC, Lee H, Shin SK, Lee SK, et al. Prognostic value of early postoperative tumor marker response in gastric cancer. Ann Surg Oncol 2013;20:3905-11.

16. Maehara Y, Kusumoto T, Takahashi I, Kakeji Y, Baba H, Akazawa K, et al. Predictive value of preoperative carcinoembryonic antigen levels for the prognosis of patients with well-differentiated gastric cancer. A multivariate analysis. Oncology 1994;51:234-7.

17. Fujimoto S, Takahashi M, Mutou T, Kobayashi K, Toyosawa T, Isawa E, et al. Improved mortality rate of gastric carcinoma patients with peritoneal carcinomatosis treated with intraperitoneal hyperthermic chemoperfusion combined with surgery. Cancer 1997;79:884-91.

18. Kumada T, Arai Y, Itoh K, Takayasu Y, Nakamura K, Ariyoshi Y, et al. Phase II study of combined administration of 5-fluorouracil, epirubicin and mitomycin-C by hepatic artery infusion in patients with liver metastases of gastric cancer. Oncology 1999;57:216-23. 